\title{
Analysis of isolates from Bangladesh highlights multiple ways to carry resistance genes in Salmonella Typhi
}

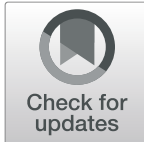

Nicholas Costa Barroso Lima ${ }^{1,2}$, Arif M. Tanmoy ${ }^{3,4,5}$, Emilie Westeel ${ }^{4}$, Luiz Gonzaga Paula de Almeida², Alain Rajoharison ${ }^{4}$, Maksuda Islam ${ }^{5}$, Hubert P. Endtz ${ }^{3,4}$, Samir K. Saha ${ }^{5,6}$, Ana Tereza Ribeiro de Vasconcelos ${ }^{2^{*+}}$ and Florence Komurian-Pradel ${ }^{4+}$

\begin{abstract}
Background: Typhoid fever, caused by Salmonella Typhi, follows a fecal-oral transmission route and is a major global public health concern, especially in developing countries like Bangladesh. Increasing emergence of antimicrobial resistance (AMR) is a serious issue; the list of treatments for typhoid fever is ever-decreasing. In addition to IncHI1-type plasmids, Salmonella genomic island (SGI) 11 has been reported to carry AMR genes. Although reports suggest a recent reduction in multidrug resistance (MDR) in the Indian subcontinent, the corresponding genomic changes in the background are unknown.

Results: Here, we assembled and annotated complete closed chromosomes and plasmids for $73 \mathrm{~S}$. Typhi isolates using short-length Illumina reads. S. Typhi had an open pan-genome, and the core genome was smaller than previously reported. Considering AMR genes, we identified five variants of SGI11, including the previously reported reference sequence. Five plasmids were identified, including the new plasmids pK91 and pK43; pK43and pHCM2 were not related to AMR. The pHCM1, pPRJEB21992 and pK91 plasmids carried AMR genes and, along with the SGI11 variants, were responsible for resistance phenotypes. pK91 also contained anr genes, conferred high ciprofloxacin resistance and was related to the H58-sublineage Bdq, which shows the same phenotype. The presence of plasmids (pHCM1 and pK91) and SGl11 were linked to two H58lineages, la and $\mathrm{Bd}$. Loss of plasmids and integration of resistance genes in genomic islands could contribute to the fitness advantage of lineage la isolates.

Conclusions: Such events may explain why lineage la is globally widespread, while the Bd lineage is locally restricted. Further studies are required to understand how these S. Typhi AMR elements spread and generate new variants. Preventive measures such as vaccination programs should also be considered in endemic countries; such initiatives could potentially reduce the spread of AMR.
\end{abstract}

Keywords: Salmonella Typhi, SGI11, Resistance genes, Typhoid fever, Bangladesh, Comparative genomics

\footnotetext{
* Correspondence: atrv@Incc.br

${ }^{\dagger}$ Ana Tereza Ribeiro de Vasconcelos and Florence Komurian-Pradel contributed equally to this work.

2Laboratório Nacional de Computação Científica, Petrópolis, Brazil

Full list of author information is available at the end of the article
}

(c) The Author(s). 2019 Open Access This article is distributed under the terms of the Creative Commons Attribution 4.0 International License (http://creativecommons.org/licenses/by/4.0/), which permits unrestricted use, distribution, and reproduction in any medium, provided you give appropriate credit to the original author(s) and the source, provide a link to the Creative Commons license, and indicate if changes were made. The Creative Commons Public Domain Dedication waiver (http://creativecommons.org/publicdomain/zero/1.0/) applies to the data made available in this article, unless otherwise stated. 


\section{Background}

Typhoid fever, a major global public health threat, is caused by Salmonella enterica serovar Typhi (S. Typhi). Due to its fecal-oral transmission route, the disease is most prevalent in the least developed regions of the world in the tropical belt, which also contains the least developed regions of the world. Over $80 \%$ of the global burden of 12 million typhoid cases per year occurs in Asia and Africa, mainly among children and adolescents [1-3]. Although the mortality rate is low $(1-2 \%)$, typhoid fever may lead to long-term physical and mental disabilities if untreated for a long time [4]. Moreover, the huge numbers of typhoid fever cases in developing countries impose a significant economic burden.

Antimicrobial therapy is the most effective treatment for typhoid fever. However, due to increasing levels of antimicrobial resistance (AMR), a small number of cases of treatment failure have been reported, even among patients treated with newer generations of antimicrobials [5-7]. Multidrug resistance (MDR) in S. Typhi-defined as co-occurring resistance to ampicillin (amp), chloramphenicol (chl) and cotrimoxazole (sxt) -was first reported in 1973 [8, 9] and resistance to ciprofloxacin (cip) emerged in the early 1990s. The list of available treatment options for typhoid fever has rapidly reduced since the emergence of AMR and treatment regimens have shifted towards quinolones. Extended-spectrum beta-lactams (e.g. ceftriaxone) or macrolides (e.g. azithromycin) are now the most effective treatment options for typhoid fever. However, extended-spectrum betalactamase (ESBL)-producing $S$. Typhi have been reported in many countries, and exhibit high levels of resistance to ceftriaxone (cro) [10-12].

In $S$. Typhi, MDR genes are usually carried by an IncHI1-type plasmid [13, 14]. However, a chromosomal Tn21-like element has recently been reported as a component of Salmonella genomic island 11 (SGI11) [15, 16]. This island has been reported to carry resistance genes for seven different antimicrobial agents, including ampicillin, chloramphenicol, and cotrimoxazole, it integrates into two or more chromosomal locations, and can confer MDR even in the absence of plasmids [16-18]. MDR has also been closely associated with the dominant haplotype H58 (genotype 4.3.1), which exhibits reduced susceptibility to quinolones [16]. Mechanisms of ciprofloxacin resistance (cip-R) usually involve chromosomal point mutations and the acquisition of AMR genes. Such mutations occur in quinolone resistance-determining regions (QRDR), which correspond to multiple locations on the DNA gyrase (gyrA and gyrB) and topoisomerase IV (parC and parE) genes [19-22]. The presence of plasmid-mediated quinolone resistance (PMQR) genes, such as $q n r$, and overexpression of efflux pump genes can also contribute to quinolone resistance $[23,24]$.
Although $\mathrm{H} 58$ is still the most prevalent MDR $S$. Typhi variant, a reduction in the frequency of isolation of H58-MDR strains has been reported. These isolates are only resistant to one or two antibiotics, i.e., ampicillin, chloramphenicol, or cotrimoxazole [25-29]. Nonetheless, the genomic changes to chromosomes or plasmids responsible for such H58 non-MDR phenotypes are yet to be described.

In this study, we generated the complete closed chromosome sequences and accessory plasmid sequences for $73 \mathrm{~S}$. Typhi strains isolated in Bangladesh between 1999 and 2013; the strains were selected for this study according to their antimicrobial resistance profile. We annotated and studied the core and pan-genomes of all isolates $(n=73)$. The genetic elements responsible for AMR (e.g. genes, mutations, genomic islands) and their locations (plasmids or chromosome) were analyzed and compared with the resistance phenotypes. We also assessed the presence, location and gene contents of SGI11 and plasmids and their associations with resistance phenotypes.

\section{Results}

\section{General genomic features and comparative genomics}

The chromosomes of all $73 \mathrm{~S}$. Typhi isolates were assembled and ranged from 4,773,823 to 4,897,593 base pairs (bp) in size (Additional file 1: Table S2). The GC content of all chromosomes was 53\%. Automatic gene annotation showed an average of 4236 (median 4230) chromosomal genes, with an average size of $643 \mathrm{bp}$ per coding sequence (Additional file 1: Table S2). The numbers of genes encoding hypothetical proteins, tRNAs, rRNAs, and pseudogenes in all isolates ranged from 871 to 949 , 75-84, 21-24, and 171-195, respectively (Additional file 1: Table S2). The lowest and highest ANIb/ANIm values were 99.89-100/99.85-99.92 respectively (Additional file 1: Table S2). The core-genome, defined as the part of the genome common to all isolates, contained 3944 genes, representing 93\% of the average gene content of the isolates (Fig. 1a). The dispensable genome (the set of genes shared by some-but not all-isolates) contained 803 genes, while the unique genome (genes present in only one isolate) contained 1855 genes (Fig. 1a). The pan-genome, corresponding to the sum of the core, dispensable and unique genomes was composed of 6602 genes. The curves for the pan and core genomes (Fig. 1b) indicated the number of core genes (green line) stabilized after the addition of the tenth genome. The pangenome fitting parameter $(\gamma=0.67$; blue line) indicates an open pan-genome [30]. Figure 1c shows the proportion of clusters of orthologous group (COG) classes for the core, dispensable and unique genomes. As the core genome represented $93 \%$ of the average number of genes in the isolates, it is reasonable that core genes make the 


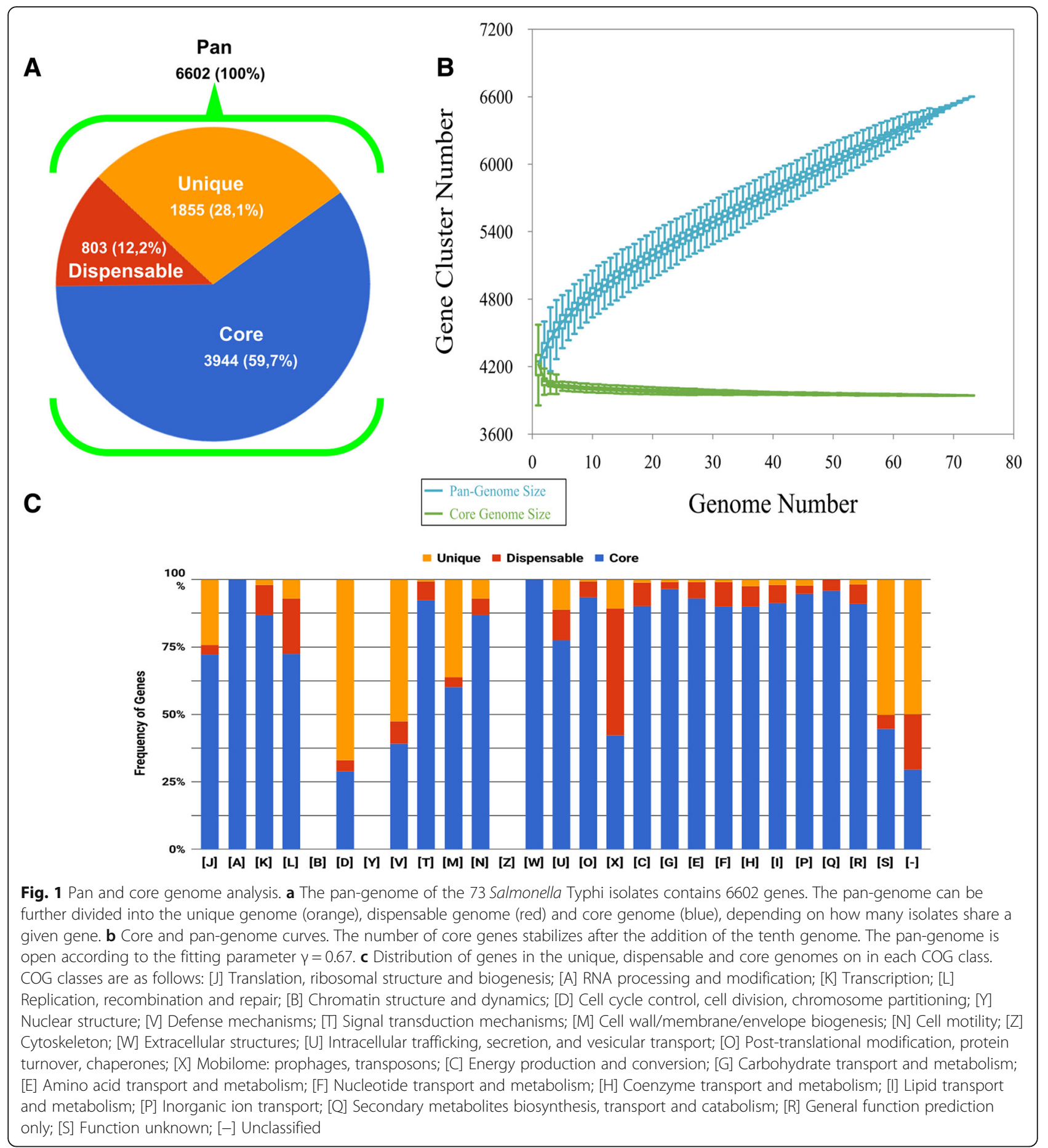

bulk of COG classes (Fig. 1c). Moreover, as typical for bacteria, none of the detected genes were related to nuclear structure (class Y); genes in COG classes B (Chromatin structure and dynamics) and $\mathrm{Z}$ (Cytoskeleton) were not detected either. A number of classes, including $\mathrm{K}$ (Transcription), L (Replication, recombination, and repair), and X (Mobilome: prophages, transposons) were represented more frequently in the dispensable genome than the unique and core genomes. We verified the location of the genes in classes $\mathrm{K}, \mathrm{L}$ and X; 49, 39 and 14\% of those genes were within prophage regions. In the unique genome set, the most common gene classes were J (Translation, ribosomal structure, and biogenesis), D (Cell cycle control, cell division, chromosome partitioning), V (Defense mechanisms) and M (Cell wall/membrane/envelope biogenesis). 
Presence of Salmonella genomic island 11 (SGI11)

Twenty-one of the 73 isolates harbored a genomic island similar to SGI11. Manual curation of gene content revealed that not all of these genomic islands were archetypical. Some isolates had the same gene content as SGI11 $(n=8)$, and we identified four variants that we named SGI11 $b(n=9)$, SGI11c $(n=1), \operatorname{SGI11} d(n=1)$ and SGI11e ( $n=2$; Fig. 2$)$.

Usually, SGI11 genomic islands contained antimicrobial resistance genes (bla $a_{\mathrm{TEM}-1}, \operatorname{cat} A 1, \operatorname{str} A, \operatorname{str} B$, sul1, sul2 and $d f r A 7$ ), mercury resistance genes (merE, merD, merA, merC, merP, merT and merR; Fig. 2a) and the qac$E \Delta 1$ gene that encodes ethidium-bromide resistance protein, a member of the small multidrug resistance (SMR) family [31]. SGI11 was previously found to interrupt the $n l p C$ or $y i d A$ gene (Chiou et al. [15]. All archetypical SGI11 in our Bangladeshi isolates disrupted the yidA gene, which encodes a sugar phosphate phosphatase. One isolate that contained the archetypical SGI11 sequence had inversion of the segment containing the $\operatorname{str} B$, strA and sul2 genes (Fig. 2a, red dotted bridge); this inversion was also observed in SGI11d (Fig. 2d). Similarly to archetypical SGI11, SGI11 $b, c$, and $d$ also disrupted the yidA gene, but contained deletions of $7857 \mathrm{bp}$ (region encoding bla $a_{\mathrm{TEM}-1}, \operatorname{str} A, \operatorname{str} B$ and $\left.s u l 2\right), 1317 \mathrm{bp}$ (region encoding catA1), or $9959 \mathrm{bp}$ (region encoding catA1, sul1, dfrA7 and qacEA1), respectively (Fig. 2b-d). SGI11e was located between the cyaY and cyaA genes, missing a 5651 bp region (encoding $\operatorname{str} B$, str $A$ and sul2) and the direction of the AMR genes and IS elements were reversed (Fig. 2e). None of the SGI11 variants interrupted the $n l p C$ gene, which has been described previously by Chiou et al. [15] as one of the probable insertion sites for the island.

\section{Plasmids}

Five different types of plasmids were detected and assembled, and ranged in size from 43,427-218,627 bp (Table 1). In total, 50 of the 73 isolates harbored plasmids: 49 isolates harbored just one type of plasmid and isolate 311189_217186 harbored two plasmids, matched (by homology) with NC_003384 and NC_003385, known as pHCM1 and pHCM2 respectively [32]; see Table 1.

Twenty pHCM1-like plasmids were assembled, and ranged in size from 214,596-218,627 bp. All pHCM1like plasmids harbored similar resistance genes as SGI11 (Fig. 2a). We also assembled 21 pHCM2-like plasmids; 17 were 106,706 bp and four were 106,705 bp, $200 \mathrm{bp}$ longer than the reference pHCM2 (NC_003385) plasmid sequence of $S$. Typhi CT18. However, the gene content of the short and long pHCM2 plasmids were the same as the reference [32].

Another plasmid, which we named pK91, was present in seven isolates. This plasmid ranged in size from 91, 848-93,445 bp, harbored the qnrS1, bla $a_{\mathrm{TEM}-1}$, sul2, tetR,

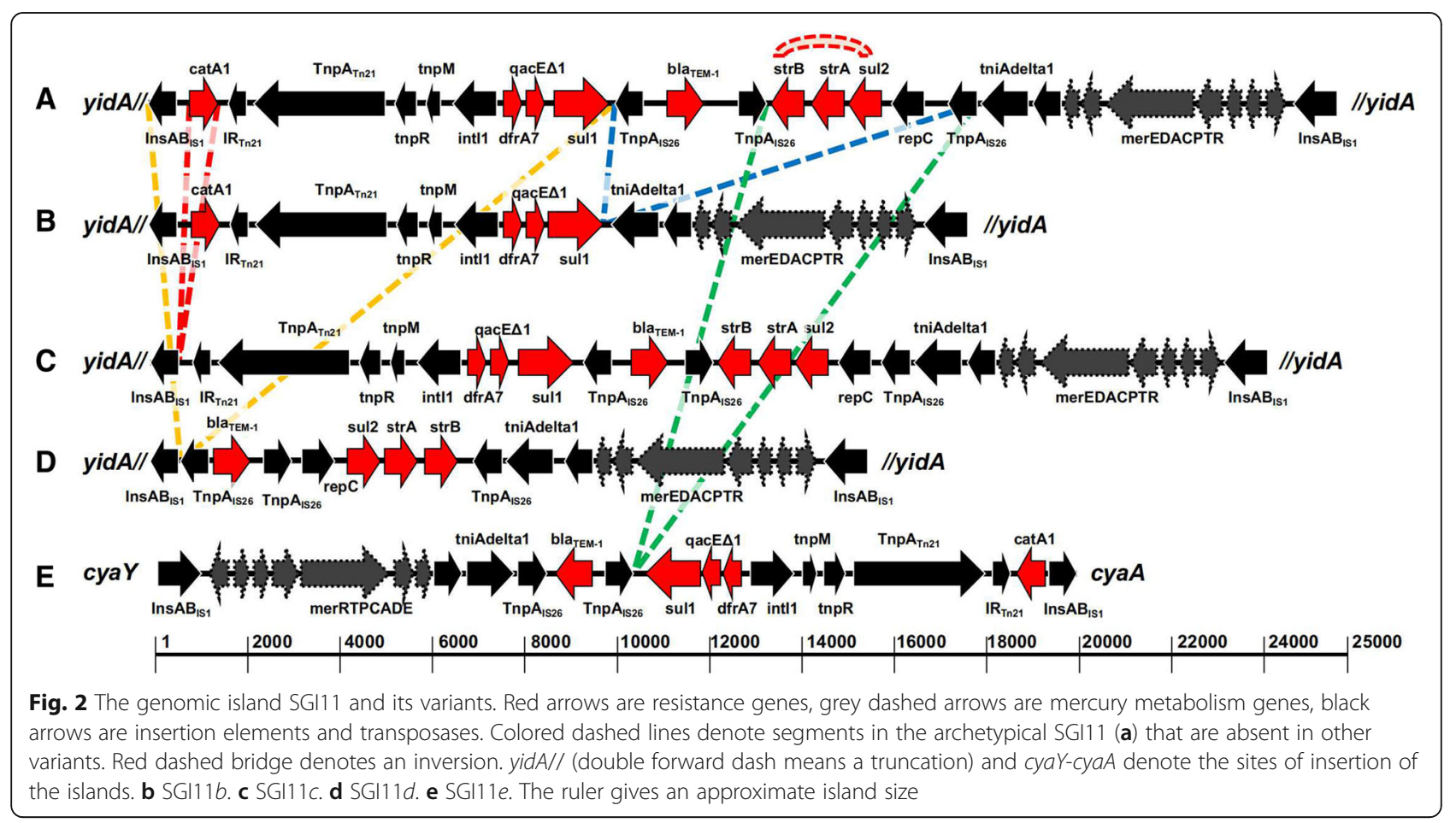


Table 1 Plasmids and genomic islands detected in the 73 S. Typhi isolates. The resistance genes present in these elements are listed

\begin{tabular}{|c|c|c|c|c|}
\hline & Name & Size range (bp) or location & Number & Resistance genes \\
\hline \multirow[t]{5}{*}{ Plasmids } & $\mathrm{pHCM1}$ & $214,596-218,627$ & 20 & bla $_{\text {TEM-1; }}$ catA1; strA; strB; sul1; sul2; dfrA7; qacE $\Delta 1$ \\
\hline & $\mathrm{pHCM} 2$ & $106,706-106,706$ & 21 & - \\
\hline & pK43 & 43,427 & 1 & - \\
\hline & PPRJEB21992 & 88,544 & 1 & bla $a_{T E M-1} ; b l a_{C T X-M-15}$ \\
\hline & pK91 & $91,848-93,445$ & 7 & $b_{\text {la }} a_{T E M-1} ;$ sul2; anrS1; tetA; tetR \\
\hline \multirow[t]{5}{*}{ Genomic Islands } & SGI11 & yidA & 8 & bla $_{\text {TEM-1; }}$ catA1; strA; strB; sul1; sul2; dfrA7; qacE $\Delta 1$ \\
\hline & SGI11b & yidA & 9 & catA1; sul1; dfrA7; qacE $\Delta 1$ \\
\hline & SGI11C & yidA & 1 & bla $_{\text {TEM-1 }} ;$ strA; strB; sul1; sul2; dfrA7; qacE $\Delta 1$ \\
\hline & SGl11d & yidA & 1 & bla $_{\text {TEM-1 }} ;$ strA; strB; sul2 \\
\hline & SGl11e & суаҮ-суаA & 2 & bla ${ }_{T E M-1} ;$ catA1; sul1; dfrA7; qacE $\Delta 1$ \\
\hline
\end{tabular}

and tet $A$ resistance genes, and shared $66 \%$ query coverage (at $99 \%$ identity in the aligned portions) with a plasmid from E. coli (CP026578). Our single ceftriaxoneresistant isolate contained an 88,544 bp plasmid, previously described as pPRJEB21992, that harbored $b l a_{\text {TEM-1 }}$ and bla $a_{\mathrm{CTX}-\mathrm{M}-15}$ [11]. Both pK91 and pPRJEB21992 contained a compendium of type IV secretion system genes. Recently, Klemm et al. [10] described a promiscuous plasmid, p60006, that confers resistance to fluoroquinolones and third-generation cephalosporins. This plasmid harbored the qurS1, bla $a_{\mathrm{TEM}-1}$ and sul2 genes (similarly to pK91) and the bla $a_{\mathrm{CTX}-\mathrm{M}-15}$ and bla $a_{\mathrm{TEM}-1}$ genes (similarly to pPRJEB21992). Figure 3a, b and c shows a comparison of the regions carrying resistance genes in p60006, pK91 and pPRJEB21992, respectively. One isolate (ID: 343077_281186) contained a 43,427 bp plasmid, which we named pK43, with high similarity to the 38
Kbp pSTY1 (CP009103) plasmid from Salmonella Typhimurium strain ATCC 13311. This plasmid lacks resistance genes, but encodes genes for pili formation and conjugation.

\section{SGI11, plasmids and comparison with genotypes and MLST}

Comparison of the SNP-based genotyping data with the presence of plasmids and SGI11 revealed few remarkable associations. Except for the three isolates (3/73) with undetermined genotype data, all isolates that carried pK91 plasmids $(n=7)$, pHCM1 plasmids $(n=20)$ or any variant of SGI11 $(n=21)$ were from genotype 4.3.1 (Haplotype 58, H58; Additional file 2: Table S3). Moreover, all isolates with SGI11 variants (19/70) belonged to H58-lineage Ia. No isolates from

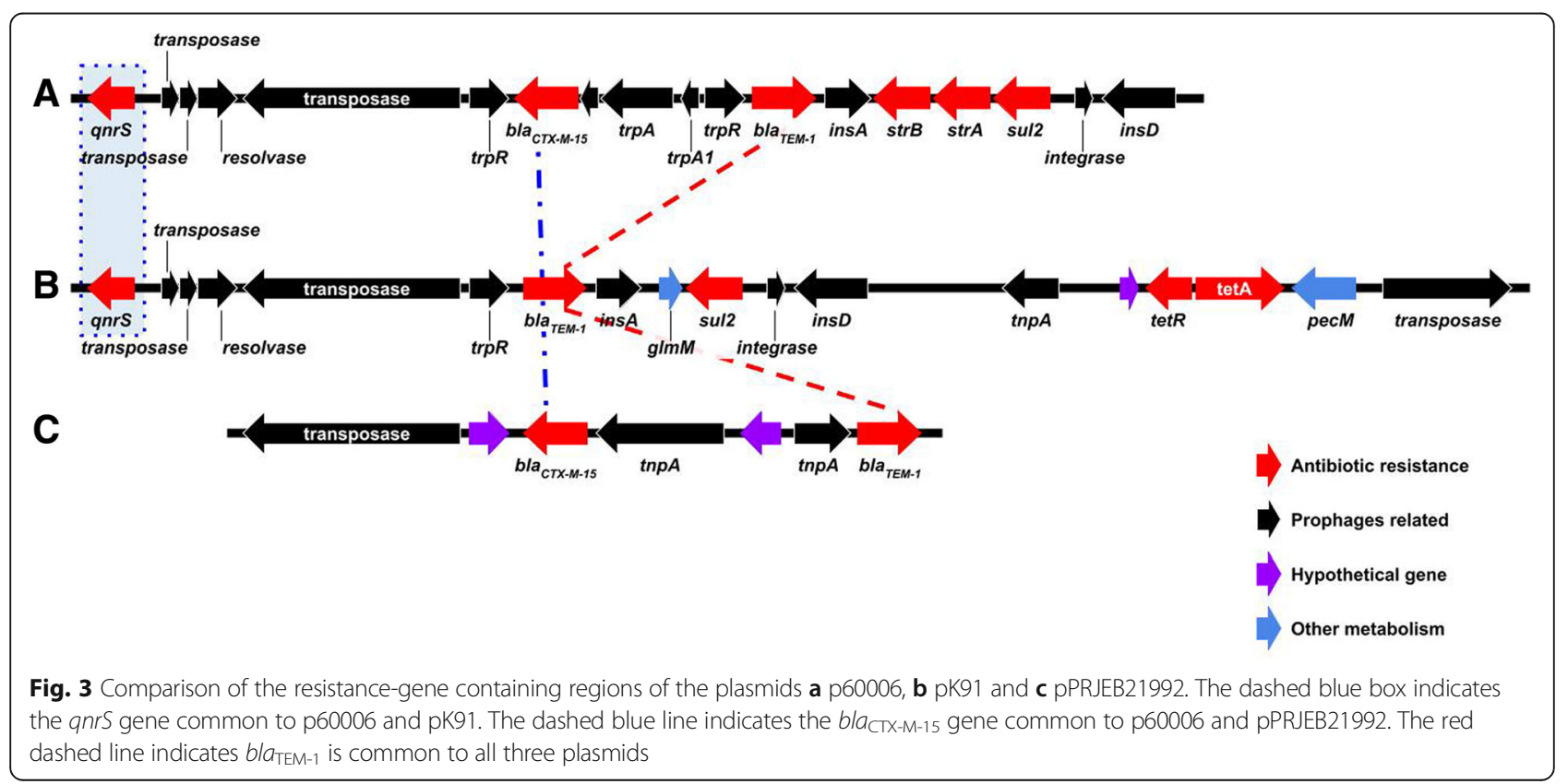




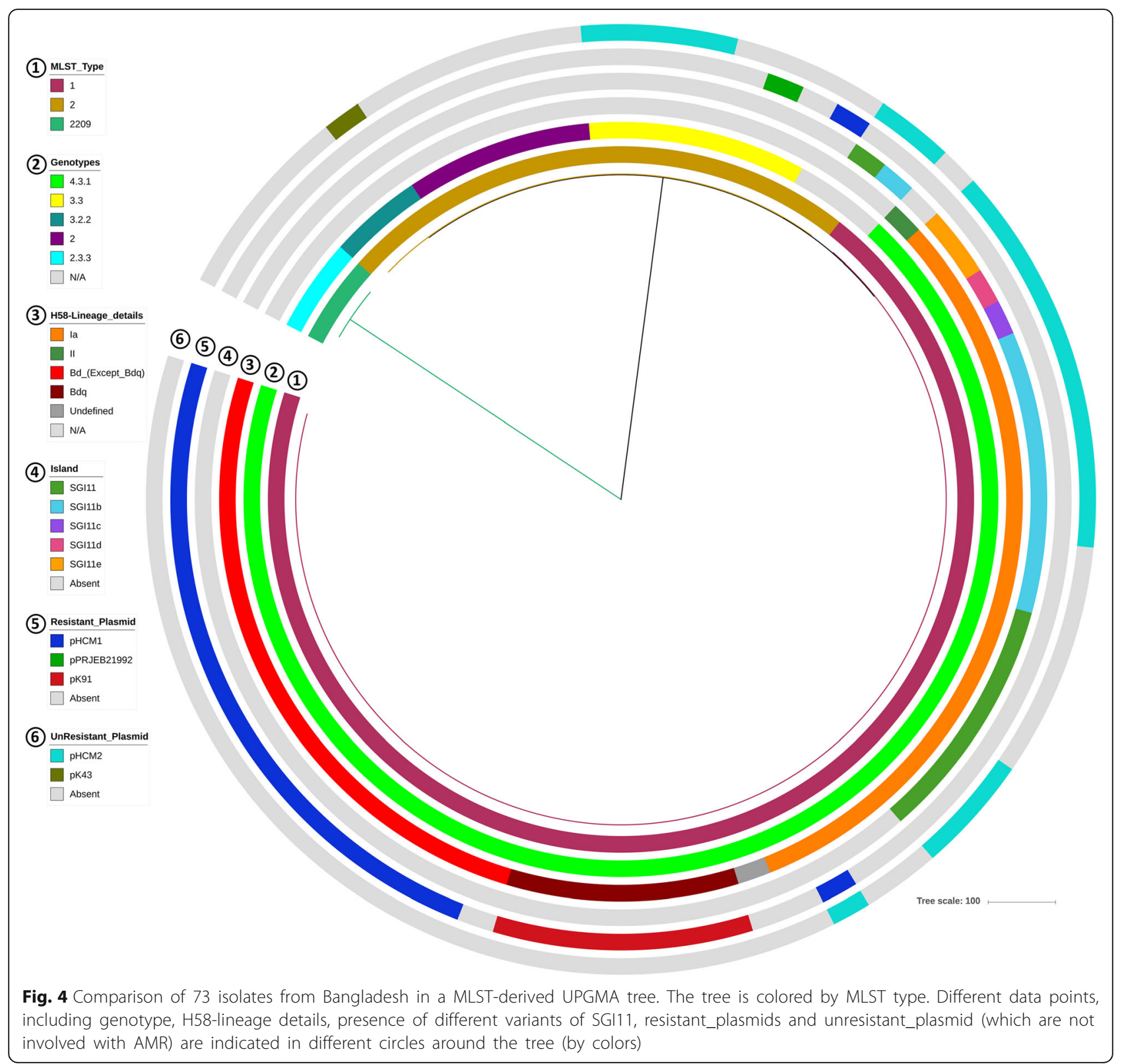

other H58 lineages or other genotypes contained SGI11 (Fig. 4, Additional file 2: Table S3).

In contrast, only one lineage Ia isolate had the pHCM1 plasmid. All other isolates with this plasmid (18/70) belonged to the newly described H58-lineage Bd; only one strain from this lineage did not contain the pHCM1 plasmid. However, none of these isolates were from the $\mathrm{Bd}$ sublineage $B d q$. In contrast, all isolates from the $B d q$ sublineage $(n=7)$ carried a pK91 plasmid (Fig. 4, Additional file 2: Table S3).

All isolates carrying pHCM2 had either genotype 3.3 $(n=4)$ or $4.3 .1(n=14)$. All isolates with the 4.3.1 genotype were from lineage Ia $(n=14)$. Only genotype 3.2.2 isolates carried the pK43 plasmid (Additional file 2: Table S3).
ST1 was the dominant MLST type among our isolates $(n=52)$, followed by ST2 $(n=18)$ and ST2209 $(n=3)$. Forty-six of the 52 ST1 isolates had either a resistance plasmid (pHCM1 or pK91) or a variant of SGI11. In contrast, only one of the 18 ST2 isolates carried pHCM1; no ST2 isolates carried pK91 and only one ST2 isolate had SGI11. None of the ST2209-type isolates had either a plasmid or SGI11.

The ceftriaxone-resistant isolate with the pPRJEB21992 plasmid had the 3.3 genotype and ST2 type.

\section{Resistance profiles}

Our 73 isolates were classified into 12 resistance profiles (Table 2), of which two were multidrug resistant (MDR) 
Table 2 Resistance profiles of the S. Typhi isolates. (R) resistant; (S) susceptible; amp, ampicillin; sxt, cotrimoxazole; chl, chloramphenicol; cip, ciprofloxacin; cro, ceftriaxone

\begin{tabular}{lll}
\hline Number of Isolates & Resistance Profile & Plasmids/Genomic Islands present \\
\hline 26 & amp-R, sxt-R, chl-R, cip-R, cro-S & pHCM1 (20); SGI11 (5) \\
15 & amp-S, sxt-S, chl-S, cip-R, cro-S & SGI11(1); pK91 (1) \\
10 & amp-S, sxt-S, chl-S, cip-S, cro-S & - \\
7 & amp-R, sxt-S, chl-S, cip-R, cro-S & SGI11d (1); pK91 (6) \\
6 & amp-S, sxt-R, chl-R, cip-R, cro-S & SGl11 (1); SGl11b (5) \\
2 & amp-S, sxt-S, chl-R, cip-R, cro-S & SGl11b (2) \\
1 & amp-R, sxt-R, chl-R, cip-S, cro-S & SGl11 (1) \\
1 & amp-R, sxt-R, chl-S, cip-R, cro-S & SGl11c (1) \\
2 & amp-R, sxt-S, chl-R, cip-R, cro-S & SGl11e (2) \\
1 & amp-R, sxt-S, chl-S, cip-S, cro-R & pPRJEB21992 (1) \\
1 & amp-S, sxt-R, chl-R, cip-S, cro-S & SGI11b (1) \\
\hline
\end{tabular}

and 10 were non-MDR (nMDR). Thirty-six of the 37 isolates with ampicillin resistance contained plasmids and/ or SGI11 variants, namely pHCM1 $(n=20$ isolates), pK91 $(n=6)$, pPRJEB21992 $(n=1)$, SGI11 $(n=6)$, SGI11c $(n=1)$, SGI11d $(n=1)$ and SGI11e $(n=2$; Table 3, Additional file 2: Table S3). Two isolates with SGI11 and one with pK91 were susceptible to ampicillin, despite carrying $b l a_{\mathrm{TEM}-1}$. Isolates with SGI1 $b$, pK43, and pHCM2 were $b l a_{\text {TEM-1 }}-$ free and susceptible to ampicillin, with one exception: one isolate harboring SGI1 $b$ without $b l a_{\text {TEM-1 }}$ was ampicillin-resistant.

Only one of the 73 isolates was resistant to ceftriaxone (Table 3, Additional file 2: Table S3). This isolate was also resistant to ampicillin, and harbors the pPRJEB21992 plasmid that encodes both bla $\mathrm{TEM}_{\mathrm{T}}$ and bla $_{\text {CTX-M-15. In terms of ciprofloxacin resistance, we }}$ identified multiple mutations in the $\operatorname{gra}$ and parC genes, including S83F, S83Y, D87G, and D87N in gyrA;

Table 3 Summary of resistance to each antibiotic tested. The genes associated with a given resistance profile, as well as the number of susceptible (S) or resistant (R) isolates, are shown. For ciprofloxacin resistance, we also show mutations on gyrA/B and parC/E genes

\begin{tabular}{|c|c|c|c|c|}
\hline & \# resistant isolates & \# susceptible isolates & $\begin{array}{l}\text { Associated resistance gene } \\
\text { (number of genes) }\end{array}$ & $\begin{array}{l}\text { gene:mutation:number of } \\
\text { isolates with mutations }\end{array}$ \\
\hline Ampicillin & 38 & 35 & bla-tem-1 (40) & Not applicable \\
\hline Ceftriaxone & 1 & 72 & bla-ctx-m-15 (1) & Not applicable \\
\hline \multirow[t]{13}{*}{ Ciprofloxacin } & 59 & 14 & qnrS1 (7) & gyrA:S83Y:29 \\
\hline & & & & gyrA:S83F:28 \\
\hline & & & & gyrA:D87G:2 \\
\hline & & & & gyrA:D87N:3 \\
\hline & & & & gyrA:N529S:6 \\
\hline & & & & gyrA:D538N:52 \\
\hline & & & & gyrB:S464F:9 \\
\hline & & & & parC:S801:1 \\
\hline & & & & parC:S80E:1 \\
\hline & & & & parC:E84K:2 \\
\hline & & & & parE:S339 L:1 \\
\hline & & & & parE:A364V:7 \\
\hline & & & & parE:L416F:1 \\
\hline Chloramphenicol & 39 & 34 & catA1 (39) & Not applicable \\
\hline Cotrimoxazole & 35 & 38 & sul1 (39); sul2 (36); dfrA7 (40) & Not applicable \\
\hline
\end{tabular}


S80I, S80R, and E84K in parC (Table 3). A quinolone resistance ( $q n r)$ gene, qnrS1 was detected in seven isolates and carried by a plasmid (herein called pK91) which also had a bla TEM-1 gene [33] (Table 3). Both gyrA and parC mutations, and the qnr genes were associated with resistance to ciprofloxacin (Additional file 2: Table S3). Other detected mutations in the non-QRDR regions include N529S, D538N of gyrA, S464F of gyrB, and S339 L, A364V and L416F of parE (Table 3).

The catA1 gene was present in PHCM1, SGI11 and the SGI11 $b$ and $e$ variants. Only one isolate with catA1 showed susceptibility to chloramphenicol. Another isolate was phenotypically resistant to chloramphenicol, but did not contain a plasmid or SGI11 carrying the catA1 gene.

Thirty-five isolates were resistant to cotrimoxazole. Of these, 27 had the three aforementioned $d f r A 1$, sul1, and sul2 genes, six had the $d f r A 1$ and sul1 genes, and one isolate only had the $d f r A 1$ gene (Table 3 , Additional file 2: Table S3). One cotrimoxazole-resistant isolate did not harbor $d f r A 1$, sul1 or sul2 genes. Thirty-eight isolates were classified as being susceptible to cotrimoxazole, 24 of these isolates did not have any of the $d f r A 1$, sul1 or sul2 genes. Eight cotrimoxazole-susceptible isolates had a sul2 gene, five had only $d f r A 1$ and sul1 genes, and one isolate had all three genes.

\section{Discussion}

In agreement with previous findings [34], all $73 S$. Typhi genomes in this study were highly conserved, as confirmed by the ANI values ( $>98 \%$; Additional file 1: Table S2) and whole genome alignments (Additional file 3: Figure S1). However, the presence or absence of SGI11-like elements led to sequence differences and variation in genome size.

The core genome analysis also indicated high genomic conservation between our 73 isolates, with 3944 genes in the $S$. Typhi core genome (60\% of the pan-genome). In contrast, a previous study of only six isolates identified 4131 core genome genes [35]. The limited geographical origin (Bangladesh) of our isolates could explain the low number of genes in the core genome. Conversely, the higher number of isolates in this study (73 vs. 6 in Baddam et al., 2015) may explain the differences in the pangenome content (6602 vs. 5426 genes). In contrast, the Salmonella enterica pan-genome has a higher gene number but smaller core genome than the $S$. Typhi isolates in this study. This difference is to be expected, as the Salmonella enterica dataset included multiple serovars [36]. Interestingly, our $S$. Typhi pan-genome contains a higher number of genes than $S$. Paratyphi-A (4670; 41 isolates) and $S$. Enteritidis (4750; 159 isolates), but a smaller number of genes than $S$. Typhimurium (7603; 47 isolates; [37]. These findings corroborate the hypothesis that the pan-genome size does not reflect the host range or ability to colonize multiple hosts [37, 38].

However, the accessory genome or serovar-specific core genome could be responsible for such host variety (Seif et al., 2018). Considering the host restriction of $S$. Typhi, the same rationale could explain why $93 \%$ of the average gene content of our isolates was present in the core genome. In contrast, serovars like $S$. Typhimurium with a variable host range have $75 \%$ of its average gene content in its core genome (3475/4661) [37]. Variation within a serovar should be reflected in the dispensable genome, whereas the unique genome should explain strain-specific characteristics. Our $S$. Typhi dispensable genome showed enrichment of COG classes X $(n=48)$, $\mathrm{L}(n=38), \mathrm{K}(n=33), \mathrm{S}(n=23$; function unknown), $\mathrm{C}$ $(n=22)$ and $\mathrm{E}(n=21)$. Class $\mathrm{X}$ refers to genes related to prophages and transposons; we found $\sim 50 \%$ of class $\mathrm{X}$ genes in the dispensable genome were located in the prophage regions of the $S$. Typhi genome. Moreover, $40 \%$ of class L (DNA repair) genes in the dispensable genome were located in prophage regions. Prophage-like elements in Salmonella serovars can play a role in recombination, contribute to virulence in the host and carry specific virulence-associated genes such as sopE. In the case of $S$. Typhi, these regions could even cause more subtle intra-serovar variation (Boyd and Brüssow 2002; Thomson et al. 2004). These prophage regions have also been reported to contribute to mechanisms of DNA repair, possibly as a part of the bacterial SOS regulatory system (Balbontín et al. 2006). Our core $S$. Typhi genome lacked a number of COG classes, including Y (genes related to nuclear structure), B (Chromatin structure and dynamics) and $\mathrm{Z}$ (Cytoskeleton). This result could be an artifact of COG class annotation, as the current version of the COG database does not include in their classes bacterial genes related to chromatin-like organization or cytoskeletal formation, even though there are only but a few genes on those related functions for bacteria. Also there may be a lack of annotation of genes related to those classes.

Remarkably, our analysis of $73 \mathrm{~S}$. Typhi genomes suggested an open pan-genome, in contrast to previous studies that reported Salmonella enterica had a closed pan-genome [36, 39]. However, these previous studies were mainly performed on the Salmonella genus, not specifically the Typhi serovar. Moreover, the low numbers of $S$. Typhi isolates in these studies could lead to discrepancies in the pan-genome results [36, 39]. An open pan-genome usually indicates bacterial species that can colonize multiple environments and exchange genetic material in multiple ways. Other more conserved species that tend to live in isolated niches with limited access to the microbial gene pool or that have a lower capacity to acquire foreign genes usually show a closed 
pan-genome [40]. Considering its human hostrestriction, $S$. Typhi should have a closed pan-genome. However, typhoid fever is endemic in many highlypopulated areas of the world, where the bacteria are transmitted through contaminated food and water. Such transmission mediums contains vast bacterial community, acting as a bacterial gene pool, which may reflect in an open pangenome [36, 39]. Moreover, prophage regions of the genome may work as hotspots for the acquisition of new genes in those regions [41]. The core/pan-genome ratio, 0.6 (3944/ 6602) in our study also points to open pangenome, as discussed previously by Rouli et al. 2015 [41]. In Addition, the number of pseudogenes in our $S$. Typhi isolates was higher; median $177(4 \%)$, similar to $\sim 200$ in $S$. Typhi CT18 reference genome, compared to $S$. Typhimurium (0.9\%) or, Escherichia coli K12 (0.7\%). A high number of pseudogenes in bacterial species could be associated with its host restriction, as it has been observed in other bacteria [34]. Certain genes required for a broad range of hosts may become pseudogenes, once bacteria get adapted to only one preferred host [34]. Hostspecificity of $S$. Typhi is mostly due to the strong selectivity of typhoid toxin for Neu5Ac- terminated glycans over Neu5Gc-terminated ones and the absence of an operational Rab32-dependent host defense pathway in human. Neu5Ac- terminated glycans are predominantly expressed in human cells, while the Neu5Gc- terminated ones are dominant in other mammals [42-44]. It could be the reason why gtgE (a cysteine protease) and sopD2 (Type III secretion system effector protein) genes became pseudogenes or missing in $S$. Typhi genome, as reported earlier $[32,42]$. Both the genes are present in other Salmonella (e.g. Typhimurium) to protect the bacteria from Rab32dependent host defense pathway in other mammalian hosts (e.g. Chimpanzees) [32, 42].

Despite high sequence conservation, we observed some differences in the resistance gene contents of our isolates, and these differences were reflected in the phenotypic resistance profiles (Table 2). This variation in resistance can be attributed to the mutations occurring on antibiotic target genes and the presence of acquired resistance genes carried on plasmids or SGI11-like islands. Different variants of SGI11, with different gene contents or orientations and locations in the chromosome, were observed among our isolates. Four of the five SGI11 variants interrupted the yidA gene of the S. Typhi genome, while SGI11e was located between the cyaY and cyaA genes (Table 1); both locations have previously been described $[15,16,18]$.

The presence of the archetypal SGI11 sequence, SGI11b, $d$ or $e$, or pHCM1 conferred chloramphenicol resistance, as these elements harbor the catA1 gene; the catA1 gene was missing from SGI11c. Only two isolates did not exhibit the corresponding resistance or susceptibility phenotypes based on the presence or absence of catA1. However, even if the catA1 gene is absent, other mechanisms such target gene mutations or the presence of an efflux pump can confer resistance [45]. In contrast, a decrease in the concentration of acetyl-CoA can inhibit the activity of catA1 and lead to a susceptibility phenotype, even in the presence of the catA1 gene [46].

Among the isolates exhibiting an ampicillin-resistant phenotype, the $b l a_{\text {TEM-1 }}$ beta-lactamase gene was present in all SGI11 variants (except variant $b$ ) and the pHCM1, pK91 and pPRJEB21992 plasmids (Figs. 2 and 3). However, three isolates were susceptible to ampicillin despite harboring the $b{ } a_{\mathrm{TEM}-1}$ resistance gene; this could be related to altered transcriptional control due to a weak bla gene promoter [47]. However, analyses of the promoter regions did not reveal any variation (data not shown). Ampicillin resistance in the absence of a bla $a_{\mathrm{TEM}-1}$ gene was also observed for one isolate, which may indicate the involvement of other resistance mechanisms like overexpression of efflux pump genes [48-50].

Unlike ampicillin and chloramphenicol, cotrimoxazole is a drug combination of trimethoprim and sulfamethoxazole, which exert a synergistic bacteriostatic effect. One mechanism of cotrimoxazole resistance involves the acquisition of folate-biosynthesis pathway genes that are resistant to the bacteriostatic effect $[51,52]$. These resistance genes can be carried by plasmids or integrons, and the combined presence of a resistant dihydropteroate synthase gene (sul1 or 2) and dihydrofolate reductase $(d f r)$ can confer resistance to cotrimoxazole. Indeed, most of our resistant isolates contained the $d f r A 7$, sul1 and sul2 genes. Other isolates had either $d f r A 7$ and sul1, or only $d f r A 7$. The sul2 gene does not confer a resistance phenotype on its own. In contrast, six of our cotrimoxazole-susceptible isolates had a $d f r A 7$ gene with a sul1 and/or sul2 gene. However, similar discrepancies were also reported from other studies who compared whole genome sequence (WGS) with antimicrobial susceptibility data [53-56].

Only one of the 73 isolates exhibited a resistance phenotype to ceftriaxone, which could be explained by the presence of a $b l a_{\text {CTX-M-15 }}$ gene on a pPRJEB21992 plasmid. A highly ceftriaxone-resistant $S$. Typhi was previously reported in Bangladesh in 1999 [57], but this isolate was not subjected to molecular characterization. Djeghout et al. [11] described the first assembled plasmid harboring $b a_{\text {CTX-M-15 }}$, pPRJEB21992, from a Bangladeshi strain isolated in 2000. We studied the same strain to compare it with other resistance plasmids we found in this study. Another plasmid, p60006, which harbors the same gene for ceftriaxone resistance was reported in a $S$. Typhi 
strain that caused an outbreak in Pakistan during 2016 and 2017 [10]. Both plasmids, pPRJEB21992 and p60006, may be the same type, but have different gene contents. Moreover, these plasmids possibly have different evolutionary origins or took different patterns of divergence, as whole-genome SNP (wgSNP) analyses revealed genotypic and phylogenetic differences between the isolates, including differences in the $b l a_{\mathrm{CTX}-\mathrm{M}-15}$ gene sequence (99\% identity and $92 \%$ coverage); [58]. Considering the origin of both of these plasmids as independent events, the chance of strains carrying any of these plasmids spreading is low, but cannot be ruled-out as both plasmids are extra-chromosomal elements. A significant increase of AMR may occur if strains carrying either of these two plasmids spread outside of their current geographical origin.

Unlike other antimicrobial agents, resistance to ciprofloxacin (cip) was common among our isolates and was associated with mutations in the $\operatorname{gyr} A / B$ and $\operatorname{par} C / E$ genes, which encode the DNA gyrase and topoisomerase IV enzymes, respectively. Indeed, 55 of the 59 cipresistant isolates contained the S83F and S83Y mutations in the gyrA gene. Another gyrA mutation, D538N was also common (52/73 isolates), but is not located in the QRDR region of the gene and should not influence susceptibility to cip [59]. Moreover, this gyrA-D538N mutation and two other mutations, gyrA-N529S and parEA364V were associated with the different genotypes of the $S$. Typhi isolates but were not involved in cip resistance [58]. Resistance can also be conferred by the $q n r$ genes [60]. Remarkably, the seven isolates with pK91 plasmids containing the qnrS1 gene had high cip MICs ( $>4.0 \mu \mathrm{g} / \mathrm{mL}$, Additional file 2: Table S3). These isolates also contained the $g y r A-S 83 Y$ mutation, but did not have other mutations in the $\operatorname{gyr} B, \operatorname{parC}$ or parE genes. The wgSNP analysis identified these isolates are part of a highly cip-resistant local H58-sublineage, Bdq [58]. S. Typhi isolates from a Pakistani outbreak also contained $q n r$ genes in the p60006 plasmid and were highly resistant to ciprofloxacin [10].

The gene contents of the p60006, pK91 and pPRJEB21992 plasmids were noticeably different (Fig. 3). However, the presence of a type IV secretion system and common IS elements in these plasmids suggest a common ancestor, and then independent patterns of divergence. The pHCM2 and pK43 plasmids had no association with resistance or other metadata (Additional file 4: Table S1 and Additional file 2: Table S3).

Surprisingly, the presence of the pHCM1 and pK91 plasmids and SGI11 islands were associated with the $S$. Typhi genotype and H58 lineage. With the exception of pPRJEB21992, all AMR-related plasmids and SGI11 were detected in isolates with genotype 4.3.1 (Haplotype 58,
H58). The seven isolates with high cip MICs that harbored the pK91 plasmid were from the newly reported H58 sublineage, Bdq (Tanmoy et al., 2018). The isolates carrying pHCM1 plasmids, which confer MDR and cip resistance, were from the local Bd lineage (but not the Bdq sublineage), while isolates with SGI11 were from lineage Ia (Additional file 2: Table S3). Thus, the local $S$. Typhi lineage, $\mathrm{Bd}$, appears to be less prone to chromosomal integration of the MDR locus than the globally widespread lineage Ia. The presence of pHCM1 and/or pK91 plasmids in lineage Bd isolates could also suggest the unaltered fitness of the lineage. Cip resistance conferred by $g y r A / B$ and $p a r C / E$ mutations did not provide any fitness advantage either, as they cannot offer any as previously reported [61]. However, the presence of gyrA/ $B$ and $p a r C / E$ mutations in 72 of our 73 isolates could indicate strong selective pressure on the genome from the overuse of antimicrobials [34].

The effect of such anthropological selective pressure could be particularly evident in Bangladesh and other South Asian countries. Self-medication and over-thecounter sale of antibiotics, especially ciprofloxacin, is prevalent in this region; ciprofloxacin has been one of the preferred treatments for enteric or diarrheal diseases since the 2000s [62, 63]. The high concentrations of this drug in meat products (chicken and livestock) can also contribute to cip resistance in the environment, leading to increased selective pressure $[64,65]$. This strong selective pressure could have played a crucial role to limit the spread of lineage $\mathrm{Bd}$ to specific geographic regions. In contrast, lineage Ia may represent the major evolutionary event of integration of the MDR locus into the chromosome to maintain the MDR phenotype, as well as a gain of fitness advantages [16].

Besides revealing these characteristics of $S$. Typhi, we obtained the complete chromosome sequences for 73 isolates, which substantially increases the number of complete (closed) chromosome sequences for this serovar available in the NCBI (only 46 sequences were available until now). However, the isolates in this study were only collected from pediatric patients as the disease is most common among school-aged children, but typhoid can occur at any age. Thus, only studying isolates from pediatric cases may not provide a complete picture of $S$. Typhi in Bangladesh. Moreover, the number of isolates in our study $(n=73)$ may be too low to detect all genetic changes that have occurred over the 15 years between 1999 and 2013, specifically chromosomal integration of the MDR locus. All of our isolates were from Bangladesh, which makes our pan-genome data relatively countryspecific. However, as a tropical country where typhoid is endemic, our core and pan-genome data should reflect the scenario of a region where $S$. Typhi is endemic. 


\section{Conclusions}

We assembled and annotated complete chromosome and plasmid sequences for $73 \mathrm{~S}$. Typhi isolates using only short-length Illumina reads. The isolates exhibited a highly conserved genome, with an open pan-genome. We report two new plasmids, pK43 with no link to resistance and pK91 that confers a high level of ciprofloxacin resistance. Multiple variants of SGI11 with different resistance genes were detected, and result in different resistance phenotypes. Plasmids carrying resistance genes were also present in many isolates with different phenotypes. The presence of SGI11 and plasmids encoding resistance genes (pHCM1 and pK91) were associated with two different H58 lineages, Ia and Bd, respectively. Shedding the plasmids and integration of the resistance genes into the genome (as islands) may have contributed to the fitness of the lineage Ia isolates; this could be one explanation for the wider geographical spread of this lineage, in comparison to the local lineage $\mathrm{Bd}$ that has remained restricted to Bangladesh. The results of this study should help us to better understand the multiple variations in the genomic elements that confer AMR in $S$. Typhi. Continuous surveillance of these elements could reveal other mechanisms by which AMR can spread in $S$. Typhi. However, preventive measures to minimize the spread of AMR should also be implemented, for example vaccination could be an effective tool to reduce the number of cases by preventing the overuse of antibiotics [66].

\section{Methods}

\section{Bacterial strains and resistance profiles}

All Salmonella Typhi isolates used in this study were collected by the Child Health Research Foundation (CHRF) from the blood of pediatric patients hospitalized at Dhaka Shishu (Children) Hospital (DSH) or pediatric outpatients treated at the Popular Diagnostic Centre (PDC) in Dhaka, Bangladesh. The CHRF team has been preserving Salmonella isolates since 1999 and currently maintains a biobank of over 3500 isolates.

Seventy three $S$. Typhi isolates were selected from the biobank based on their antimicrobial resistance phenotype (Table 2). We re-confirmed the identity of all strains using standard biochemical tests and agglutination tests with specific antisera for Salmonella species (Thermo Scientific, Waltham, MA, USA). Antimicrobial susceptibility to ampicillin (amp), cotrimoxazole (sxt) and chloramphenicol (chl) were determined using disk diffusion assays (Oxoid; Thermo Scientific). Brothmicrodilution was employed to determine the minimum inhibitory concentrations (MIC) for ciprofloxacin (cip) and ceftriaxone (cro) (Sigma Aldrich, St. Louis, MO, USA). All zone diameters and MIC data were interpreted according to EUCAST v8, 2018 ([67], 2018).
Metadata for all 73 isolates (sample, organism, year of isolation, setting, patient age [months] and sex) are presented in Additional file 4: Table S1.

\section{DNA sequencing, genome assembly, genome annotation and comparative genomics}

Isolates were cultured on MacConkey agar (Oxoid, Thermo Scientific) overnight, checked for visible contamination (and re-plated if any contamination was observed), and all colonies were picked and suspended in water. QIAamp DNA Mini Kits (Qiagen, Hilden, Germany) were used to extract DNA from the suspensions on the same day. Whole genome sequencing (WGS) was performed using an Illumina-HiSeq 4000 platform, generating $2 \times 150 \mathrm{bp}$ paired-end reads with an average coverage of $121 \mathrm{x}$, at The Oxford Genomics Centre of the Wellcome Trust Centre for Human Genetics, Oxford, UK.

Quality assessment of sequencing reads was conducted using FastQC [68]. Due to the high-quality scores of the reads and absence of adapter sequences, quality and read trimming were deemed unnecessary. Paired-end reads were first assembled using Newbler v3.0 [69]. More than 99.37\% of assembled bases for all isolates had Q40 or more, as calculated using Newbler. We used JSpecies [70] to calculate the average nucleotide identity (ANI) with BLAST ([ANIb]; [71] and Mummer ([ANIm]; [72] for all isolates plus Salmonella enterica subsp. enterica serovar Typhi str. CT18 (accession NC_003198.1). ANIb/ANIm of our isolates in comparison to $S$. Typhi CT18 were higher than $99,85 \%$ which allowed scaffolds to be aligned against S. Typhi CT18 using cross_match [73] to create a layout of ordered and oriented scaffolds to be concatenated with gaps between scaffolds estimated from the alignment and filled with 'N'. The layout of scaffolds was subjected to manual curation in order to 1) verify any missing scaffolds on the alignment; 2) confirm gap sizes; and 3) confirm the expected number of repeated scaffolds in agreement with their read coverage estimated by Newbler. This curated superscaffold was subjected to gap filling in two steps. In the first step, we used GapFiller v1.11 [74]. In step 2, remaining intraand inter-scaffold gaps that were not closed by GapFiller v1.11 were locally assembled using Newbler v3.0. Reads present at both ends of a gap were selected and assembled. Contigs obtained this way and that spanned the gap and anchored on consensus sequences on both sides were added to the consensus, thus filling the gap. Step 2 was done manually to each remaining gap from step 1 and subjected to confirmation of each closed gap to avoid erroneous gap filling. Scaffolds that did not align to the chromosome of $S$. Typhi CT18 were aligned to the $n t$ NCBI database in order to verify if they had plasmid origin. Those with plasmid origin were assembled 
using the approach described previously. Visual aid to the assembly process can be found in the Additional file 5: Figure S2. A similar approach was previously used to close a Klebsiella genomes [75]. The SABIA pipeline was used for automated annotation [76]. Assembled sequences were submitted to the NCBI (accession IDs are shown in Additional file 4: Table S1).

During the superscaffold formation step, the presence of a Salmonella genomic island (SGI) was noticed in 21 strains, due to careful manual curation of the superscaffold formation step. This island carries several resistance genes and is not present in the reference $S$. Typhi genome. Based on sequence similarity and gene content, the SGI was identified as Salmonella genomic island 11 ([SGI11] accession number KM023773; [15]. According to Chiou et al. [15], SGI11 can be located at two chromosomal positions, interrupting the yidA gene or $n l p C$ gene. Contigs were aligned against the SGI11, $n l p C$ and $y i d A$ sequences to determine the presence of the island and its position of insertion. For strains in which there was evidence of a SGI11 element but neither yidA nor $n l p C$ were interrupted, the extremities of the contigs harboring the segments of the island were locally assembled and the gene neighborhood was determined after annotation.

To observe conserved synteny blocks, we aligned all isolates and S. Typhi CT18 using Progressive Mauve [77]. We used RPS-BLAST, a variant of PSI-BLAST [78] to identify proteins with homology to COG protein profiles in the NCBI database at an e-value of 0.001. The best hit was selected and COG cluster classification was transferred to the protein query. Pan and core genome analysis was performed using PGAP v1.2.1 [79]. Coding sequences, protein sequences and COG classification for all isolates were used as input, along with an e-value of $1 \mathrm{e}-10$ at $70 \%$ identity and similarity. All protein sequences were aligned against each other using blastall and the resulting output was imported into MCL [80], a part of PGAP, to cluster the genes. After clustering, PGAP computes the pan and core genomes by strain combination from 1 to $n$ strains, where $n$ denotes the maximum number of strains ( $n=73$ in this study). Heap's law and an exponential law were employed to fit the pan and core genomes, respectively [79]. PanGP was used to plot the pan and core genome curves [81].

After assembly and annotation of the chromosomes and plasmids, AMR genes on the SGI11 and plasmids were manually annotated. We also used Abricate [82] to corroborate the AMR genes using the following databases: Resfinder, ARG-ANNOT, CARD, NCBI Bacterial Antimicrobial Resistance Reference Gene Database, EcOH, PlasmidFinder, VFDB and Ecoli_VF. We verified prophage regions on the chromosome and plasmids with PHASTER [83] to verify the presence of some classes of dispensable genome genes on these elements.
We previously obtained genotype data for 70 of the 73 isolates in this study [58]. The multilocus sequence typing (MLST) data for the isolates was determined using Enterobase [84]. The MLST data was used to build a UPGMA phylogenetic tree. The Euclidean distance matrix and UPGMA tree were computed using the dist and hclust functions, respectively, of the $\mathrm{R}$ stats package. The tree was displayed and annotated using the online version of iTOL v4 [85].

\section{Additional files}

Additional file 1: Table S2. Sheet 1. General information about the isolates assembled. Sheet 2. Average Nucleotide Identity calculated by BLAST. Sheet 3. Average Nucleotide Identity calculated by MUMMER. (XLSX $71 \mathrm{~kb}$ )

Additional file 2: Table S3. Ampicilin, ciprofloxacin, chloramphenicol, ceftriaxone, and cotrimoxazole resistance. (XLSX 50 kb)

Additional file 3: Figure S1. Isolate chromosomes were aligned with ProgressiveMauve. Conserved sequence block can be observed as they are marked with the same color. (JPEG 4269 kb)

Additional file 4: Table S1. Sheet NCBI ID: Accession numbers for sequences deposited on NCBI. Sheet Metadata: metadata for the isolates studied. (XLSX $15 \mathrm{~kb}$ )

Additional file 5: Figure S2. Schematic summary of the assembly and gap filling process. (JPG $256 \mathrm{~kb}$ )

\section{Abbreviations}

amp: Ampicillin; AMR: Antimicrobial resistance; ANI: Average nucleotide identity; ANIb: Average nucleotide identity with blast; ANIm: Average nucleotide identity with MUMMER; bp: Base pairs; chl: Chloramphenicol; CHRF: Child Health Research Foundation; cip: Ciprofloxacin; COG: Cluster of orthologous groups; cro: Ceftriaxone; DSH: Dhaka Shishu (children) Hospital; ESBL: Extended-spectrum beta-lactamase; GC: Guanine and cytosine; Kbp: Kilobase pairs; Mbp: Megabase pairs; MDR: Multidrug resistance; MIC: Minimum inhibitory concentration; MLST: Multilocus sequence typing; NCBI: National Center for Biotechnology Information; nMDR: Non-multidrug resistant; PDC: Popular Diagnostic Centre; PMQR: Plasmid-mediated quinolone resistance; Q40: Quality score 40; QRDR: Quinolone resistance determining region; -R: Resistant; -S: Susceptible; SGI: Salmonella genomic island; SMR: Small multidrug resistance; ST1: Sequence type 1; ST2: Sequence type 2; sxt: Cotrimoxazole; WGS: Whole genome sequence; wgSNP: Whole genome single nucleotide polymorphism

\section{Acknowledgements}

The authors sincerely thank Mr. Mohammad Saiful Islam Sajib and Mr. Hafizur Rahman for their assistance with the antimicrobial susceptibility tests. We thank the High-Throughput Genomics Group at the Wellcome Trust Centre for Human Genetics (funded by Wellcome Trust grant reference 090532/Z/09/Z) for generation of the sequencing data.

\section{Authors' contributions}

SKS, FKP, HPE conceived the project. ATRV coordinated the Brazilian team. AMT and MI performed bacterial culture, antibiotic susceptibility tests, and DNA extraction. AMT and AR prepared DNA and sequencing shipment, DNA aliquot management for sequencing. NCBL and LGPA performed sequence assembly and annotation. NCBL performed the pan/core genome analysis. NCBL performed the SGl and plasmid analysis with contributions from EW and AMT. AMT performed the lineage analysis. NCBL, EW and AMT wrote the draft with contributions from ATRV, FKP, LGPA, HPE and SKS. All authors read and approved the final manuscript.

\section{Funding}

This study was financially supported by the European COMPARE project (http://www.compare-europe.eu/) under the European Union's Horizon 2020 
research and innovation programme, grant agreement No. 643476 and CAPES process number 23038.010041/2013-13. This study was also financed in part by the Coordenação de Aperfeiçoamento de Pessoal de Nível Superior-Brasil (CAPES) under Finance Code 001. The funders had no role in data collection and analysis, decision to publish or preparation of the manuscript.

Arif M Tanmoy received an Allocations de Recherche pour une Thèse au Sud (ARTS) scholarship from the Institut de Recherche pour le Développement (IRD), France, to participate in this project for his PhD thesis.

The funding bodies did not take part in the design of the study and collection, analysis, and interpretation of data and in writing the manuscript.

\section{Availability of data and materials}

Sequence and annotation data that support the findings of this study have been deposited in GenBank with the accession codes being listed in Additional file 4: Table S1.

\section{Ethics approval and consent to participate}

Not applicable.

\section{Consent for publication}

Not applicable.

\section{Competing interests}

The authors declare that they have no competing interests.

\section{Author details}

'Departamento de Bioquímica e Biologia Molecular, Universidade Federal do Ceará, Fortaleza, Ceará, Brazil. ${ }^{2}$ Laboratório Nacional de Computação Científica, Petrópolis, Brazil. ${ }^{3}$ Department of Medical Microbiology \& Infectious Diseases, Erasmus MC, Rotterdam, the Netherlands. ${ }^{4}$ Fondation Mérieux - Laboratoire des Pathogènes Emergents, Lyon, France. ${ }^{5}$ Child Health Research Foundation, Department of Microbiology, Dhaka Shishu Hospital, Dhaka 1207, Bangladesh. 'Bangladesh Institute of Child Health, Dhaka Shishu Hospital, Dhaka 1207, Bangladesh.

\section{Received: 13 February 2019 Accepted: 20 June 2019} Published online: 28 June 2019

\section{References}

1. Brusch JL, Garvey T, Corales R, Schmitt SK. Typhoid fever: background, pathophysiology, epidemiology. Medscape. 2018; https://emedicine. medscape.com/article/231135-overview. Accessed 10 Apr 2018.

2. Chau TT, Campbell Jl, Galindo CM, Van Minh Hoang N, Diep TS, Nga TT, et al. Antimicrobial drug resistance of Salmonella enterica serovar typhi in asia and molecular mechanism of reduced susceptibility to the fluoroquinolones. Antimicrob Agents Chemother. 2007:51:4315-23.

3. GBD 2016 Disease and Injury Incidence and Prevalence Collaborators. Global, regional, and national incidence, prevalence, and years lived with disability for 328 diseases and injuries for 195 countries, 1990-2016: a systematic analysis for the Global Burden of Disease Study 2016. Lancet. 2017;390:1211-59.

4. Crump JA, Luby SP, Mintz ED. The global burden of typhoid fever. Bull World Health Organ. 2004;82:346-53.

5. Pham Thanh D, Karkey A, Dongol S, Ho Thi N, Thompson CN, Rabaa MA, et al. A novel ciprofloxacin-resistant subclade of H58 Salmonella Typhi is associated with fluoroquinolone treatment failure. Elife. 2016;5:e14003.

6. Arjyal A, Basnyat B, Nhan HT, Koirala S, Giri A, Joshi N, et al. Gatifloxacin versus ceftriaxone for uncomplicated enteric fever in Nepal: an open-label, two-centre, randomised controlled trial. Lancet Infect Dis. 2016;16:535-45.

7. Butt T, Ahmad RN, Mahmood A, Zaidi S. Ciprofloxacin treatment failure in typhoid fever case, Pakistan. Emerg Infect Dis. 2003;9:1621-2.

8. Olarte J, Galindo E. Salmonella typhi resistant to chloramphenicol, ampicillin, and other antimicrobial agents: strains isolated during an extensive typhoid fever epidemic in Mexico. Antimicrob Agents Chemother. 1973;4:597-601.

9. Mirza SH, Beeching NJ, Hart CA. Multi-drug resistant typhoid: a global problem. J Med Microbiol. 1996:44:317-9.

10. Klemm EJ, Shakoor S, Page AJ, Qamar FN, Judge K, Saeed DK, et al. Emergence of an extensively drug-resistant Salmonella enterica serovar Typhi clone harboring a promiscuous plasmid encoding resistance to fluoroquinolones and third-generation cephalosporins. MBio. 2018;9. https:// doi.org/10.1128/mBio.00105-18.

11. Djeghout B, Saha S, Sajib MSI, Tanmoy AM, Islam M, Kay GL, et al. Ceftriaxone-resistant Salmonella Typhi carries an Incl1-ST31 plasmid encoding CTX-M-15. J Med Microbiol. 2018; http://jmm. microbiologyresearch.org/content/journal/jmm/10.1099/jmm.0.000727.

12. Godbole GS, Day MR, Murthy S, Chattaway MA, Nair S. First report of CTXM-15 Salmonella Typhi from England. Clin Infect Dis. 2018. https://doi.org/ 10.1093/cid/ciy032.

13. Phan M-D, Kidgell C, Nair S, Holt KE, Turner AK, Hinds J, et al. Variation in Salmonella enterica serovar typhi IncHI1 plasmids during the global spread of resistant typhoid fever. Antimicrob Agents Chemother. 2009;53:716-27.

14. Holt KE, Phan MD, Baker S, Duy PT, Nga TVT, Nair S, et al. Emergence of a globally dominant IncHI1 plasmid type associated with multiple drug resistant typhoid. PLoS Negl Trop Dis. 2011;5:e1245.

15. Chiou C-S, Alam M, Kuo J-C, Liu Y-Y, Wang P-J. Chromosome-mediated multidrug resistance in Salmonella enterica serovar Typhi. Antimicrob Agents Chemother. 2015;59:721-3.

16. Wong VK, Baker S, Pickard DJ, Parkhill J, Page AJ, Feasey NA, et al. Phylogeographical analysis of the dominant multidrug-resistant H58 clade of Salmonella Typhi identifies inter- and intracontinental transmission events. Nat Genet. 2015;47:632-9.

17. Chiou C-S, Lauderdale T-L, Phung DC, Watanabe H, Kuo J-C, Wang P-J, et al. Antimicrobial resistance in Salmonella enterica serovar Typhi isolates from Bangladesh, Indonesia, Taiwan, and Vietnam. Antimicrob Agents Chemother. 2014;58:6501-7.

18. Hendriksen RS, Leekitcharoenphon P, Lukjancenko O, Lukwesa-Musyani C, Tambatamba B, Mwaba J, et al. Genomic signature of multidrug-resistant Salmonella enterica serovar typhi isolates related to a massive outbreak in Zambia between 2010 and 2012. J Clin Microbiol. 2015;53:262-72.

19. Chen S, Cui S, McDermott PF, Zhao S, White DG, Paulsen I, et al. Contribution of target gene mutations and efflux to decreased susceptibility of Salmonella enterica serovar typhimurium to fluoroquinolones and other antimicrobials. Antimicrob Agents Chemother. 2007;51:535-42.

20. Gaind R, Paglietti B, Murgia M, Dawar R, Uzzau S, Cappuccinelli P, et al. Molecular characterization of ciprofloxacin-resistant Salmonella enterica serovar Typhi and Paratyphi A causing enteric fever in India. J Antimicrob Chemother. 2006:58:1139-44.

21. Hirose K, Hashimoto A, Tamura K, Kawamura Y, Ezaki T, Sagara H, et al. DNA sequence analysis of DNA gyrase and DNA topoisomerase IV quinolone resistance-determining regions of Salmonella enterica serovar Typhi and serovar Paratyphi A. Antimicrob Agents Chemother. 2002;46:3249-52.

22. Menezes GA, Harish BN, Khan MA, Goessens WHF, Hays JP. Antimicrobial resistance trends in blood culture positive Salmonella Typhi isolates from Pondicherry, India, 2005-2009. Clin Microbiol Infect. 2012;18:239-45.

23. Pazhani GP, Chakraborty S, Fujihara K, Yamasaki S, Ghosh A, Nair GB, et al. QRDR mutations, efflux system \& antimicrobial resistance genes in enterotoxigenic Escherichia coli isolated from an outbreak of diarrhoea in Ahmedabad, India. Indian J Med Res. 2011;134:214-23.

24. Sjölund-Karlsson M, Howie R, Rickert R, Newton A, Gonzalez-Aviles G, Crump JA. Plasmid-mediated quinolone resistance in isolates of Salmonella enterica serotype Typhi, USA. Int J Antimicrob Agents. 2015;45:88-90.

25. Singhal L, Gupta PK, Kale P, Gautam V, Ray P. Trends in antimicrobial susceptibility of Salmonella Typhi from North India (2001-2012). Indian J Med Microbiol. 2014;32:149-52.

26. Das S, Samajpati S, Ray U, Roy I, Dutta S. Antimicrobial resistance and molecular subtypes of Salmonella enterica serovar Typhi isolates from Kolkata, India over a 15 years period 1998-2012. Int J Med Microbiol. 2017;307:28-36.

27. Dutta S, Das S, Mitra U, Jain P, Roy I, Ganguly SS, et al. Antimicrobial resistance, virulence profiles and molecular subtypes of Salmonella enterica serovars Typhi and Paratyphi A blood isolates from Kolkata, India during 2009-2013. PLoS One. 2014;9:e101347.

28. Zellweger RM, Basnyat B, Shrestha P, Prajapati KG, Dongol S, Sharma PK, et al. A 23-year retrospective investigation of Salmonella Typhi and Salmonella Paratyphi isolated in a tertiary Kathmandu hospital. PLoS Negl Trop Dis. 2017:11:e0006051.

29. Chand HJ, Rijal KR, Neupane B, Sharma VK, Jha B. Re-emergence of susceptibility to conventional first line drugs in Salmonella isolates from enteric fever patients in Nepal. J Infect Dev Ctries. 2014;8:1483-7.

30. Tettelin H, Riley D, Cattuto C, Medini D. Comparative genomics: the bacterial pan-genome. Curr Opin Microbiol. 2008;11:472-7. 
31. Bay DC, Rommens KL, Turner RJ. Small multidrug resistance proteins: a multidrug transporter family that continues to grow. Biochim Biophys Acta. 2008;1778:1814-38.

32. Parkhill J, Dougan G, James KD, Thomson NR, Pickard D, Wain J, et al. Complete genome sequence of a multiple drug resistant Salmonella enterica serovar Typhi CT18. Nature. 2001;413:848-52.

33. Hopkins KL, Wootton L, Day MR, Threlfall EJ. Plasmid-mediated quinolone resistance determinant qnrS1 found in Salmonella enterica strains isolated in the UK. J Antimicrob Chemother. 2007;59:1071-5.

34. Holt KE, Parkhill J, Mazzoni CJ, Roumagnac P, Weill F-X, Goodhead I, et al. High-throughput sequencing provides insights into genome variation and evolution in Salmonella Typhi. Nat Genet. 2008:40:987-93.

35. Baddam R, Kumar N, Shaik S, Lankapalli AK, Ahmed N. Genome dynamics and evolution of Salmonella Typhi strains from the typhoid-endemic zones. Sci Rep. 2015;4:7457.

36. Jacobsen A, Hendriksen RS, Aaresturp FM, Ussery DW, Friis C. The Salmonella enterica pan-genome. Microb Ecol. 2011;62:487-504.

37. Seif Y, Kavvas E, Lachance JC, Yurkovich JT. Genome-scale metabolic reconstructions of multiple Salmonella strains reveal serovar-specific metabolic traits. Nature. 2018; https://www.nature.com/articles/s41467-01806112-5.

38. Mclnerney JO, McNally A, O'Connell MJ. Why prokaryotes have pangenomes. Nat Microbiol. 2017;2:17040

39. Gordienko EN, Kazanov MD, Gelfand MS. Evolution of pan-genomes of Escherichia coli, Shigella spp., and Salmonella enterica. J Bacteriol. 2013;195: 2786-92.

40. Medini D, Donati C, Tettelin H, Masignani V, Rappuoli R. The microbial pangenome. Curr Opin Genet Dev. 2005;15:589-94.

41. Rouli $L$, Merhej V, Fournier P-E, Raoult D. The bacterial pangenome as a new tool for analysing pathogenic bacteria. New Microbes New Infect. 2015;7: 72-85. https://doi.org/10.1016/j.nmni.2015.06.005.

42. Galán JE. Typhoid toxin provides a window into typhoid fever and the biology of Salmonella Typhi. Proc Natl Acad Sci U S A. 2016;113:6338-44.

43. Deng L, Song J, Gao X, Wang J, Yu H, Chen X, et al. Host adaptation of a bacterial toxin from the human pathogen Salmonella Typhi. Cell. 2014;159: 1290-9.

44. Spanò S, Galán JE. A Rab32-dependent pathway contributes to Salmonella typhi host restriction. Science. 2012;338:960-3.

45. Schwarz S, Kehrenberg C, Doublet B, Cloeckaert A. Molecular basis of bacterial resistance to chloramphenicol and florfenicol. FEMS Microbiol Rev. 2004;28:519-42.

46. Potrykus J, Wegrzyn G. Chloramphenicol-sensitive Escherichia coli strain expressing the chloramphenicol acetyltransferase (cat) gene. Antimicrob Agents Chemother. 2001;45:3610-2.

47. Tristram SG. Novel blaTEM-positive ampicillin-susceptible strains of Haemophilus influenzae. J Infect Chemother. 2009;15:340-2.

48. Okusu $H$, Ma D, Nikaido H. AcrAB efflux pump plays a major role in the antibiotic resistance phenotype of Escherichia coli multiple-antibioticresistance (Mar) mutants. J Bacteriol. 1996:178:306-8.

49. Padilla E, Llobet E, Doménech-Sánchez A. Klebsiella pneumoniae AcrAB efflux pump contributes to antimicrobial resistance and virulence. Antimicrob Agents Chemother. 2010; http://aac.asm.org/content/54/1/ 177.short.

50. Piddock LJV. Multidrug-resistance efflux pumps - not just for resistance. Nat Rev Microbiol. 2006;4:629-36

51. Munita JM, and Arias CA. Mechanisms of antibiotic resistance. In Virulence mechanisms of bacterial pathogens, Fifh Edition (eds Kudva, I. T. et al.) 481511 (American Society of Microbiology, 2016).

52. Schmitz F-J, Perdikouli M, Beeck A, Verhoef J, Fluit AC. Resistance to trimethoprim-sulfamethoxazole and modifications in genes coding for dihydrofolate reductase and dihydropteroate synthase in European Streptococcus pneumoniae isolates. J Antimicrob Chemother. 2001;48:935-6.

53. Kagambèga A, Lienemann T, Frye JG, Barro N, Haukka K. Whole genome sequencing of multidrug-resistant Salmonella enterica serovar Typhimurium isolated from humans and poultry in Burkina Faso. Trop Med Health. 2018:46:4.

54. McDermott PF, Tyson GH, Kabera C, Chen Y, Li C, Folster JP, et al. Wholegenome sequencing for detecting antimicrobial resistance in nontyphoidal Salmonella. Antimicrob Agents Chemother. 2016;60:5515-20.

55. Neuert S, Nair S, Day MR, Doumith M, Ashton PM, Mellor KC, et al. Prediction of phenotypic antimicrobial resistance profiles from whole genome sequences of non-typhoidal Salmonella enterica. Front Microbiol. 2018;9:592.

56. Nguyen M, Long SW, McDermott PF, Olsen RJ, Olson R, Stevens RL, et al. Using machine learning to predict antimicrobial MICs and associated genomic features for nontyphoidal Salmonella. J Clin Microbiol. 2019;57. https://doi.org/10.1128/JCM.01260-18.

57. Saha SK, Talukder SY, Islam M, Saha S. A highly ceftriaxone-resistant Salmonella typhi in Bangladesh. Pediatr Infect Dis J. 1999;18:387.

58. Tanmoy AM, Westeel E, De Bruyne K, Goris J, Rajoharison A, Sajib MSI, et al. Salmonella enterica serovar Typhi in Bangladesh: exploration of genomic diversity and antimicrobial resistance. MBio. 2018;9. https://doi.org/10.1128/ mBio.02112-18.

59. Bagel S, Hüllen V, Wiedemann B, Heisig P. Impact of gyrA and parC mutations on quinolone resistance, doubling time, and supercoiling degree of Escherichia coli. Antimicrob Agents Chemother. 1999;43:868-75.

60. Redgrave LS, Sutton SB, Webber MA. Fluoroquinolone resistance: mechanisms, impact on bacteria, and role in evolutionary success. Trends Microbiol. 2014; https://www.sciencedirect.com/science/article/pii/ S0966842X14000894.

61. Baker S, Duy PT, Nga TVT, Dung TTN, Phat W, Chau TT, et al. Fitness benefits in fluoroquinolone-resistant Salmonella Typhi in the absence of antimicrobial pressure. Elife. 2013;2:e01229.

62. Biswas M, Roy MN, Manik MIN. Self medicated antibiotics in Bangladesh: a cross-sectional health survey conducted in the Rajshahi City. BMC Public Health. 2014; https://bmcpublichealth.biomedcentral.com/articles/10.1186/ 1471-2458-14-847.

63. Biswas M, Roy DN, Tajmim A, Rajib SS, Hossain M, Farzana F, et al. Prescription antibiotics for outpatients in Bangladesh: a cross-sectional health survey conducted in three cities. Ann Clin Microbiol Antimicrob. 2014;13:15.

64. Sattar S, Hassan MM, Islam SKM, Alam M. Antibiotic residues in broiler and layer meat in Chittagong district of Bangladesh. Veterinary. 2014; https:// core.ac.uk/download/pdf/25688270.pdf.

65. Chowdhury S, Hassan MM, Alam M, Sattar S, Bari MS, Saifuddin AKM, et al. Antibiotic residues in milk and eggs of commercial and local farms at Chittagong, Bangladesh. Vet World. 2015;8:467-71.

66. Lipsitch M, Siber GR. How can vaccines contribute to solving the antimicrobial resistance problem? MBio. 2016;7. https://doi.org/10.1128/ mBio.00428-16.

67. EUCAST. Breakpoint tables for interpretation of MICs and zone diameters: The European Committee on Antimicrobial Susceptibility Testing. http:// www.eucast.org

68. Andrews S. FastQC: a quality control tool for high throughput sequence data; 2010.

69. Margulies M, Egholm M, Altman WE, Attiya S, Bader JS, Bemben LA, et al. Genome sequencing in microfabricated high-density picolitre reactors. Nature. 2005:437:376-80.

70. Richter M, Rosselló-Móra R. Shifting the genomic gold standard for the prokaryotic species definition. Proc Natl Acad Sci U S A. 2009;106:19126-31.

71. Altschul SF, Gish W, Miller W, Myers EW, Lipman DJ. Basic local alignment search tool. J Mol Biol. 1990;215:403-10.

72. Kurtz S, Phillippy A, Delcher AL, Smoot M, Shumway M, Antonescu C, et al Versatile and open software for comparing large genomes. Genome Biol. 2004:5:R12.

73. http://www.phrap.org. http://www.phrap.org/phredphrapconsed.html. Accessed 10 Dec 2018.

74. Boetzer M, Pirovano W. Toward almost closed genomes with GapFiller. Genome Biol. 2012;13:R56.

75. Nicolás MF, Ramos PIP, Marques de Carvalho F, Camargo DRA, de Fátima Morais Alves C, Loss de Morais $\mathrm{G}$, et al. Comparative genomic analysis of a clinical isolate of subsp., a KPC-2 and OKP-B-6 beta-lactamases producer harboring two drug-resistance plasmids from Southeast Brazil. Front Microbiol. 2018;9:220.

76. Almeida LGP, Paixão R, Souza RC, da Costa GC, Barrientos FJA, dos Santos MT, et al. A system for automated bacterial (genome) integrated annotation--SABIA. Bioinformatics. 2004;20:2832-3.

77. Darling AE, Mau B, Perna NT. progressiveMauve: multiple genome alignment with gene gain, loss and rearrangement. PLoS One. 2010;5:e11147.

78. Altschul SF, Madden TL, Schäffer AA, Zhang J, Zhang Z, Miller W, et al. Gapped BLAST and PSI-BLAST: a new generation of protein database search programs. Nucleic Acids Res. 1997;25:3389-402. 
79. Zhao Y, Wu J, Yang J, Sun S, Xiao J, Yu J. PGAP: pan-genomes analysis pipeline. Bioinformatics. 2012;28:416-8.

80. van Dongen SM. Graph clustering by flow simulation; 2000.

81. Zhao Y, Jia X, Yang J, Ling Y, Zhang Z, Yu J, et al. PanGP: a tool for quickly analyzing bacterial pan-genome profile. Bioinformatics. 2014;30:1297-9.

82. Seemann T. ABRicate, mass screening of contigs for antimicrobial resistance or virulence genes; 2017.

83. Arndt D, Grant JR, Marcu A, Sajed T, Pon A, Liang Y, et al. PHASTER: a better, faster version of the PHAST phage search tool. Nucleic Acids Res. 2016;44: W16-21.

84. EnteroBase. http://enterobase.warwick.ac.uk/species/index/senterica. Accessed 5 Dec 2018.

85. Letunic I, Bork P. Interactive tree of life (iTOL) v3: an online tool for the display and annotation of phylogenetic and other trees. Nucleic Acids Res. 2016;44:W242-5.

\section{Publisher's Note}

Springer Nature remains neutral with regard to jurisdictional claims in published maps and institutional affiliations.

Ready to submit your research? Choose BMC and benefit from:

- fast, convenient online submission

- thorough peer review by experienced researchers in your field

- rapid publication on acceptance

- support for research data, including large and complex data types

- gold Open Access which fosters wider collaboration and increased citations

- maximum visibility for your research: over $100 \mathrm{M}$ website views per year

At BMC, research is always in progress.

Learn more biomedcentral.com/submissions 